\title{
SNR Enhancement of OTDR Using Biorthogonal Codes and Generalized Inverses
}

\author{
Duckey Lee, Student Member, IEEE, Hosung Yoon, Pilhan Kim, Jonghan Park, Na Young Kim, and \\ Namkyoo Park, Member, IEEE
}

\begin{abstract}
We propose and experimentally demonstrate a novel technique which significantly improves the performance of optical time-domain reflectometry (OTDR). Applying biorthogonal codes and Moore-Penrose generalized inverses, a signal-to-noise ratio enhancement of up to $9.04 \mathrm{~dB}$, namely a coding gain, was achieved over conventional OTDR.
\end{abstract}

Index Terms-Biorthogonal codes, Moore-Penrose generalized inverses, optical fiber communications, optical time-domain reflectometry (OTDR).

\section{INTRODUCTION}

$\mathbf{O}$ PTICAL time-domain reflectometry (OTDR) characterizes optical fibers, by injecting an optical probe pulse into the fiber under test and detecting the backscattered optical signals including Rayleigh backscattering and Fresnel reflections. Increasing the pulsewidths of probe pulses improves the signal-to-noise ratio (SNR) of the detected signal and accordingly the dynamic/measurement range of OTDR, but degrades the spatial resolution. In order to overcome this tradeoff between SNR and the resolution, various coding techniques have been suggested [1]-[4]. In principle, these methods utilize coded sequences of unit short pulse to obtain a higher trace level and, thus, better SNR from the increased effective pulsewidth/power for the probe, and at the same time to keep the spatial resolution of the short unit pulse. The final OTDR trace, in conventional shape but with improved SNR, can then be restored from the coded traces by decoding processes. Still, to the best of our knowledge, experimental demonstration of SNR improvement using the coding technique has been limited to the complementary correlation OTDR based on the Golay codes [3], which suffer from the resolution penalty (or an equivalent $3-\mathrm{dB}$ reduction in the SNR improvement at the same level of spatial resolution) inherently associated with the decoding process of the correlation OTDR [5].

In this letter, we show that it is possible to avoid this penalty by preserving the shape of the unit pulse in the decoding process with a proper code set and decoding method based on the linear matrix operation (basically composed of addition and subtraction). Experimental verification shows an SNR enhancement over conventional OTDR of close to $9 \mathrm{~dB}$ at a code length of 256 , in excellent agreement with the derived coding gain formula.

Manuscript received May 12, 2004; revised August 17, 2004.

The authors are with Optical Communication Systems Laboratory, School of Electrical Engineering and Computer Science, Seoul National University, Seoul 151-742, Korea (e-mail: nkpark@plaza.snu.ac.kr).

Digital Object Identifier 10.1109/LPT.2004.836916

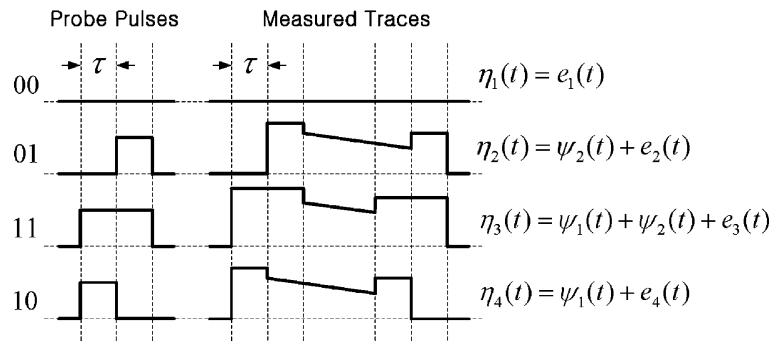

Fig. 1. Example of probe pulse sequences with 2-bit biorthogonal code set, and an illustration of traces measured with each codeword.

\section{THEORY}

For the linear matrix operation, we employ the biorthogonal code set [6] for the OTDR pulse coding and its generalized inverse for the decoding process, which is known to yield minimum least-square errors in the estimation of linearly transformed objects [7]. When the code length is $n$, a biorthogonal code set consists of $2 n$ codewords constituting a $2 n \times n$ biorthogonal code matrix $\mathbf{B}$. The $n$-bit biorthogonal code matrix $\mathbf{B}_{m}$ can be constructed from the Hadamard matrix $\mathbf{H}_{m}$ as in (1), where the code length $n$ has the form of $\left(n=2^{m}\right.$, $m=1,2, \ldots)$ and $\overline{\mathbf{H}}_{m}$ is the complement of $\mathbf{H}_{m}$ [6]

$$
\mathbf{B}_{m}=\left(\begin{array}{c}
\mathbf{H}_{m} \\
\overline{\mathbf{H}}_{m}
\end{array}\right), \mathbf{H}_{m}=\left(\begin{array}{cc}
\mathbf{H}_{m-1} & \mathbf{H}_{m-1} \\
\mathbf{H}_{m-1} & \overline{\mathbf{H}}_{m-1}
\end{array}\right), \mathbf{H}_{1}=\left(\begin{array}{cc}
0 & 0 \\
0 & 1
\end{array}\right) \text {. }
$$

To apply such a code set for the measurement, we probe the fiber under test with the $n$-bit coded pulse sequences, for all the constructed $2 n$ codewords. The final OTDR trace in conventional shape can then be restored from the $2 n$ measured traces, using the generalized inverse $\mathbf{B}^{+}=\left(\mathbf{B}^{T} \mathbf{B}\right)^{-1} \mathbf{B}^{T}$, where $\mathbf{B}^{T}$ and $\mathbf{B}^{-1}$ mean the transpose and the inverse of matrix $\mathbf{B}$, respectively [7].

Fig. 1 shows a simplest example of such an implementation using a 2-bit biorthogonal code set which has four codewords. Here, $\psi_{1}(t)$ and $\psi_{n}(t)$ represent an ideal (noiseless) conventional OTDR trace obtained by launching a unit pulse into the fiber and the time-delayed version of $\psi_{1}(t)$, i.e., $\psi_{n}(t)=$ $\psi_{1}(t-(n-1) \tau)$, respectively, where $\tau$ is the pulsewidth of the unit pulse. For this arrangement, $\eta_{i}(t)$, the trace measured with the $i$ th codeword, and $e_{i}(t)$, the noise amplitude added in each measurement, take the following matrix relation:

$$
\left(\begin{array}{l}
\eta_{1}(t) \\
\eta_{2}(t) \\
\eta_{3}(t) \\
\eta_{4}(t)
\end{array}\right)=\mathbf{B}\left(\begin{array}{c}
\psi_{1}(t) \\
\psi_{2}(t)
\end{array}\right)+\left(\begin{array}{c}
e_{1}(t) \\
e_{2}(t) \\
e_{3}(t) \\
e_{4}(t)
\end{array}\right), \quad \mathbf{B}=\left(\begin{array}{ll}
0 & 0 \\
0 & 1 \\
1 & 1 \\
1 & 0
\end{array}\right)
$$


In order to recover the final OTDR trace $\psi_{1}(t)$ from the measured traces, we need to multiply the generalized inverse matrix $\mathbf{B}^{+}$by the matrix of the measured traces as shown in (3), where $\widehat{\psi}_{i}(t)$ represents the estimate of $\psi_{i}(t)$

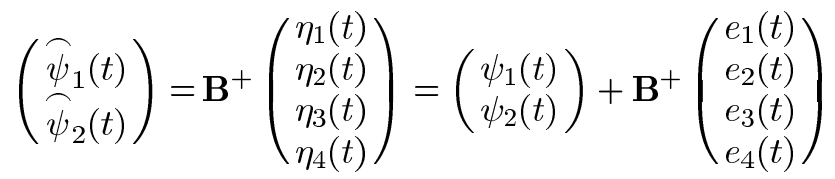

$$
\begin{aligned}
& \mathbf{B}^{+}=\frac{1}{3}\left(\begin{array}{cccc}
0 & -1 & 1 & 2 \\
0 & 2 & 1 & -1
\end{array}\right) \\
& \left(\begin{array}{c}
\overbrace{1}(t) \\
\hat{\psi}_{2}(t)
\end{array}\right)=\mathbf{B}^{+}\left(\begin{array}{c}
0 \\
\eta_{2}(t) \\
\eta_{2}(t)+\eta_{4}(t) \\
\eta_{4}(t)
\end{array}\right) \\
& =\left(\begin{array}{c}
\psi_{1}(t) \\
\psi_{2}(t)
\end{array}\right)+\mathbf{B}_{\mathrm{eff}}^{+}\left(\begin{array}{c}
e_{1}(t) \\
e_{2}(t) \\
e_{3}(t) \\
e_{4}(t)
\end{array}\right) \\
& \mathbf{B}_{\mathrm{eff}}^{+}=\frac{1}{3}\left(\begin{array}{llll}
0 & 0 & 0 & 3 \\
0 & 3 & 0 & 0
\end{array}\right) \text {. }
\end{aligned}
$$

Meanwhile, it is evident from Fig. 1 that measurements for $\eta_{1}(t)$ and $\eta_{3}(t)$ can be neglected because $\eta_{1}(t)$ is a simple noise component and $\eta_{3}(t)$ is the linear summation of $\eta_{2}(t)$ and $\eta_{4}(t)$. For the case of code length $n$, we can also omit two measurements, $\eta_{1}(t)$ and $\eta_{n+1}(t)$, by using $\eta_{1}(t)=0$ and $\eta_{n+1}(t)=$ $\eta_{n / 2+1}(t)+\eta_{3 n / 2+1}(t)$. By applying this simplification to (3), the estimates of the time-shifted traces, $\widehat{\psi}_{i}(t)$ 's can be obtained from only two measurements rather than four, as shown in (4). Note that the matrix which is effectively applied to the noise is modified from $\mathbf{B}^{+}$to $\mathbf{B}_{\text {eff }}^{+}$, as $\eta_{3}(t)$ is substituted with $\eta_{2}(t)+$ $\eta_{4}(t)$. As a result, the trace estimates $\widehat{\psi}_{i}(t)$ 's can be obtained

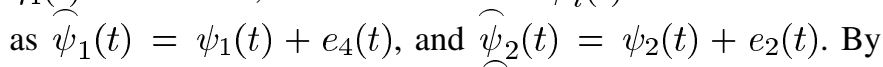
averaging the obtained estimates $\widetilde{\psi}_{i}(t)$ 's after reversely timeshifting them by $(i-1)$ multiples of $\tau$, we can obtain the final trace with the maximum SNR

$$
\frac{\widetilde{\psi}_{1}(t)+{\widehat{\psi_{2}}}_{2}(t+\tau)}{2}=\psi_{1}(t)+\frac{e_{4}(t)+e_{2}(t+\tau)}{2} .
$$

The previously described coding-decoding algorithm can be easily extended to the general $n$-bit cases. The $(2 n \times 1)$ column vector of measured coded traces $\boldsymbol{\eta}(t)$ at time $t$ can be expressed with the linear model $\boldsymbol{\eta}(t)=\mathbf{B} \boldsymbol{\psi}(t)+\mathbf{e}(t)$, where $\mathbf{B}, \boldsymbol{\psi}(t)$, and $\mathbf{e}(t)$ are $(2 n \times n)$ matrix of the biorthogonal code set, $(n \times 1)$ column vector of the time-delayed traces, and $(2 n \times 1)$ column vector of the noise, respectively. The decoding process can then be written as $\widehat{\boldsymbol{\psi}}(t)=\mathbf{B}^{+} \boldsymbol{\eta}(t)$, where $\widehat{\boldsymbol{\psi}}(t)$ and $\mathbf{B}^{+}$are $(n \times 1)$ column vector of the trace estimates and $(n \times 2 n)$ matrix of the generalized inverse, respectively. For this general case, the final trace can then be obtained as

$\frac{1}{n} \sum_{i=1}^{n} \widehat{\psi}_{i}(t+(i-1) \tau)=\psi_{1}(t)+\frac{1}{n} \sum_{i=1}^{n} \sum_{j=1}^{2 n} b_{i, j}^{+} e_{j}(t+(i-1) \tau)$

where $b_{i, j}^{+}$represents an $(i, j)$ element of the matrix $\mathbf{B}_{\mathrm{eff}}^{+}$. The coding gain (SNR enhancement over conventional trace averaging OTDR) then can be obtained by calculating the ratio of

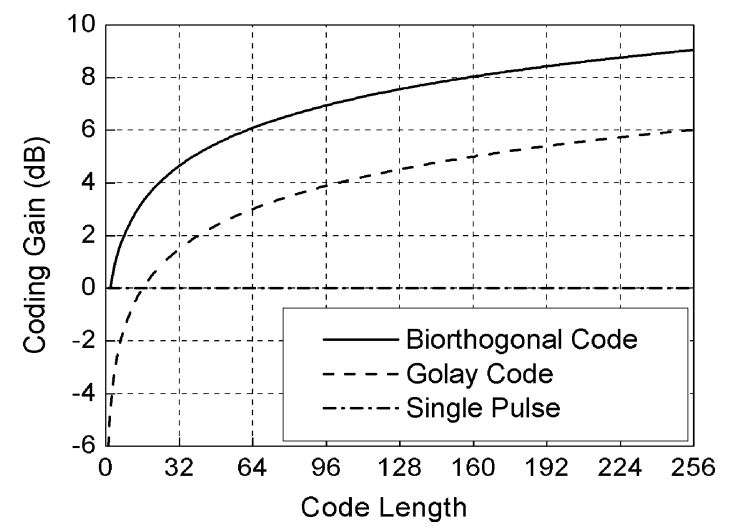

Fig. 2. Comparison of coding gains obtained with the biorthogonal code, the Golay code, and the conventional single-pulse method.

noise amplitude between the coding OTDR and conventional OTDR, as expressed in (8). The noise power in the finally processed trace using $n$-bit biorthogonal codes also can be derived from the coefficients of noise components in (6). Using the following equation which gives the noise power in each trace estimate $\widehat{\psi}_{i}(t)$ :

$$
\sum_{j=1}^{2 n}\left(b_{i, j}^{+}\right)^{2}=\frac{2 n^{3}+2 n^{2}+12}{n^{2}(n+1)^{2}}, \quad i=1, \ldots, n .
$$

The coding gain at the code length $n$ then can be written as

$$
\frac{\sqrt{\frac{\sigma^{2}}{2 n-2}}}{\sqrt{\frac{2 n^{3}+2 n^{2}+12}{n^{3}(n+1)^{2}} \sigma^{2}}}=\sqrt{\frac{n^{3}(n+1)^{2}}{4(n-1)\left(n^{3}+n^{2}+6\right)}}
$$

under the assumption that the noise $e_{i}(t)$ 's are uncorrelated, zero-mean random processes with variance $\sigma^{2}$. In deriving (8), it is worthwhile to note that we used $N=2 n-2$ in the calculation of the averaged noise power of conventional OTDR, as we leave out two measurements $\eta_{1}(t)$ (null) and $\eta_{n+1}(t)$ (full) from the $2 n$ codewords. Fig. 2 compares the coding gains of the biorthogonal and the Golay codes at the same spatial resolution, as a function of the code length. As can be seen from the figure, the coding gain of the biorthogonal code is superior to that of the Golay code by more than $3 \mathrm{~dB}$ at all code lengths.

\section{EXPERIMENTS}

To experimentally verify our proposed technique, we developed an in-house OTDR board capable of modulating the probe pulses according to the biorthogonal codes (Fig. 3). A pulsed-type Fabry-Pérot laser diode and an avalanche photodiode (APD) were used for the optical source and detector, respectively. The backscattered optical signals from the fiber link were redirected to the APD, using an optical circulator. A trans-impedance amplifier was added behind the APD, to convert currents from the APD into voltages adjusted to the dynamic range of the analog-to-digital converter (ADC). The ADC sampled the incoming voltages at $10 \mathrm{MHz}$, and the sampled traces were transmitted to the personal computer (PC), where the restoration process of the final trace was executed. Texas Instrument's (TI) TMS320VC33 was used as a digital 


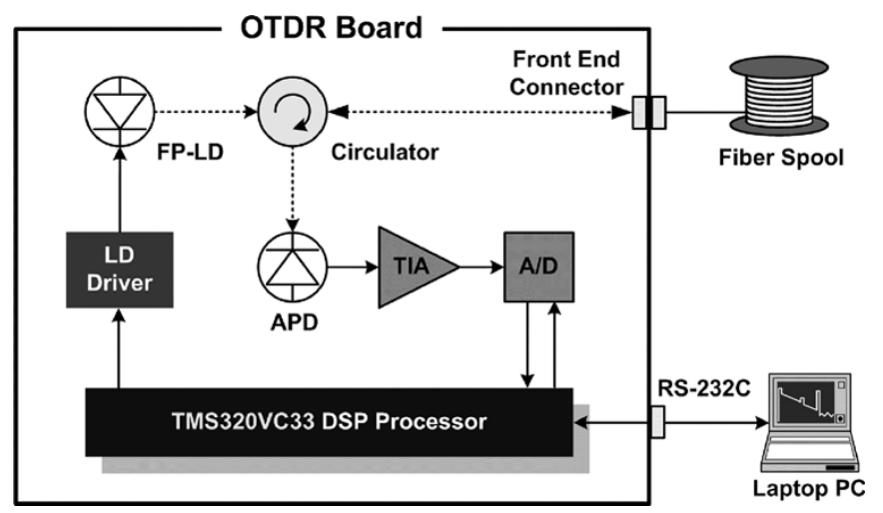

Fig. 3. Experimental setup.

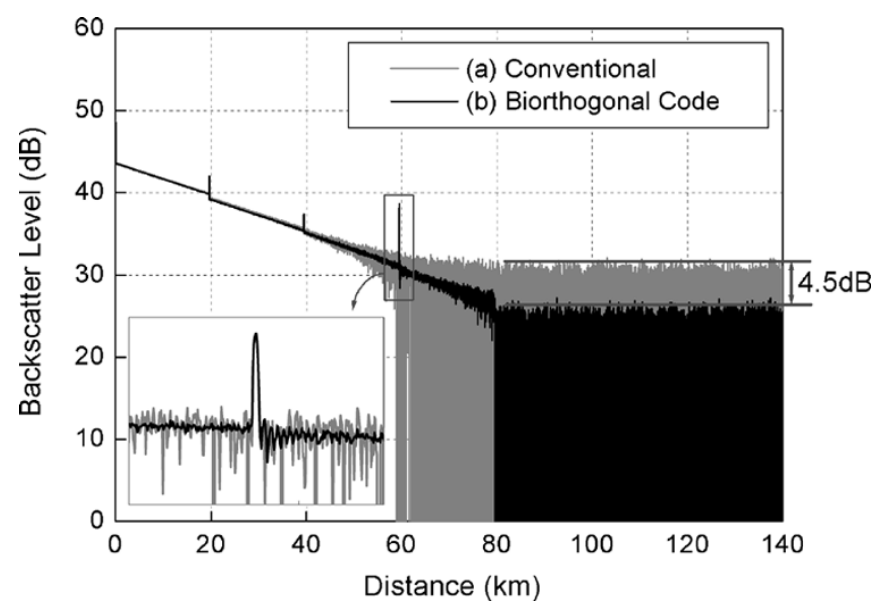

Fig. 4. Final OTDR trace obtained from (a) the conventional single-pulse averaging method, and (b) the proposed coding-decoding processes with biorthogonal codes.

signal processor to take charge of pulse coding, trace acquisition, averaging, and data communications. It is noteworthy that, for the complete trace recovery at the PC, the traces were transmitted in the linear scale using the 32-bit TI floating point mode, not in the log scale commonly used by conventional OTDR.

For a specific experiment, four spools of 20-km single-mode fiber and a laptop PC were connected to the board. Fig. 4(a) shows the OTDR trace in conventional mode, directly obtained from the simple average of 510 single-pulsed traces [Fig. 5(a)]. To compare, Fig. 4(b) illustrates the final OTDR trace in coded mode, obtained from the decoding process of 510 traces measured with a 256-bit biorthogonal code set [Fig. 5(b)]. For both of the measurement mode (conventional, biorthogonal), the pulsewidth of the unit probe pulse (or bit duration) was $500 \mathrm{~ns}$, corresponding to a spatial resolution of about $50 \mathrm{~m}$. As can be clearly seen, the SNR achieved with the

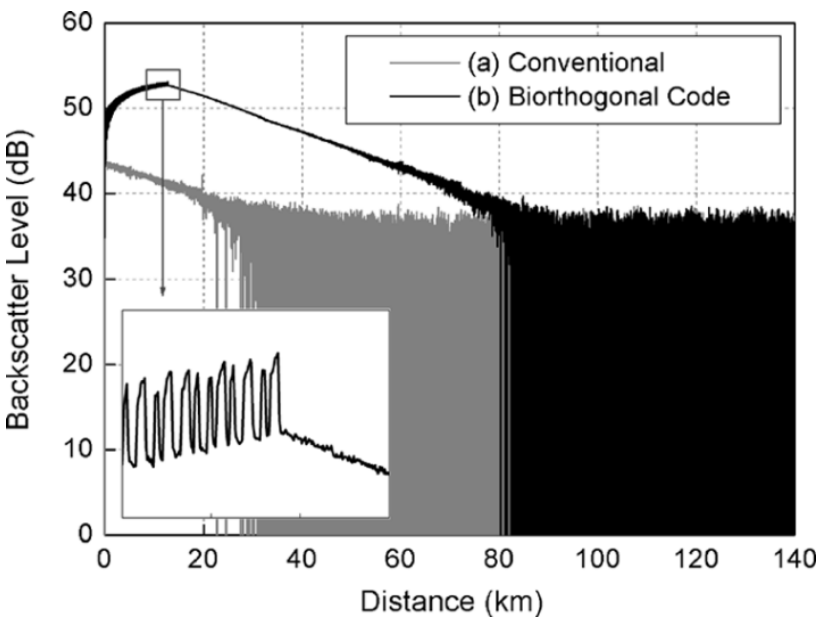

Fig. 5. Intermediate raw trace data measured with (a) the conventional single pulse, and (b) the pulse sequences coded with one of the 510 codewords (256-bit code set), respectively.

suggested coding-decoding processes was significantly better than the SNR achieved with an OTDR in the conventional averaging mode, while the same measurement time was spent in both cases. At the code length of 256 , the measured one-way dynamic range enhancement was about $4.5 \mathrm{~dB}$ (Fig. 4), in excellent agreement with the two-way coding gain value of $9.04 \mathrm{~dB}$ which was theoretically predicted from (8) and Fig. 2.

\section{CONCLUSION}

We have proposed a novel coding-decoding technique based on the linear operations of biorthogonal codes, for SNR enhancement of noncoherent OTDR. Experimental results demonstrated that the proposed technique produced an SNR improvement of up to $9 \mathrm{~dB}$, in excellent agreement with the developed theoretical analysis.

\section{REFERENCES}

[1] K. Okada and K. Hashimoto, "Optical cable fault location using correlation technique,” Electron. Lett., vol. 16, pp. 629-630, 1980.

[2] P. Healey, "Optical orthogonal pulse compression codes by hopping," Electron. Lett., vol. 17, pp. 970-971, 1981.

[3] M. Nazarathy, S. A. Newton, R. P. Giffard, D. S. Moberly, F. Sischka, W. R. Trutna Jr., and S. Foster, "Real-time long range complementary correlation optical time domain reflectometer," J. Lightw. Technol., vol. 7, pp. 24-38, Jan. 1989.

[4] M. Jones, "Using simplex codes to improve OTDR sensitivity," IEEE Photon. Technol. Lett., vol. 15, pp. 822-824, Jul. 1993.

[5] Y. Gong and A. D. Stokes, "Resolution of correlation optical time domain reflectometry," in CLEO/PACIFIC RIM, 1995, Paper P97, pp. 303-304.

[6] B. Sklar, Digital Communications. Englewood Cliffs, NJ: PrenticeHall, 1988.

[7] S. L. Campbell and C. D. Meyer Jr., Generalized Inverses of Linear Transformations. New York: Pitman, 1979. 